\title{
Towards a new treatment against polymicrobial infections: high antibacterial activity of lemon IntegroPectin against Pseudomonas aeruginosa and Escherichia coli
}

Alessandro Presentato, ${ }^{[\mathrm{ac}]}$ Antonino Scurria,${ }^{[\mathrm{b}]}$ Lorenzo Albanese,${ }^{[\mathrm{c}]}$ Pasquale Picone ${ }^{[\mathrm{d}]}$ Mario Pagliaro, ${ }^{[\mathrm{b}]}$ Federica Zabini, ${ }^{[\mathrm{cc}}$ Francesco Meneguzzo, ${ }^{[\mathrm{c}]}$ Rosa Alduina, ${ }^{[\mathrm{a}] *}$ Domenico Nuzzo, ${ }^{\left[\mathrm{d}{ }^{*}\right.}$ Rosaria Ciriminna ${ }^{[\mathrm{b}] *}$

Besides causing frequent infections of surgical sites, and of chronic decubitus ulcers, $P$. aeruginosa is also involved in infections of the urinary tract, ${ }^{[3]}$ and in cystic fibrosis promoting accelerated decline of pulmonary function. Unfortunately, the bacterium exhibits significant resistance to both innate immune anti-microbial peptides and to several antibiotics. ${ }^{[4]}$

The increase of antibiotic resistant phenotypes and antibiotic resistance genes in the environment ${ }^{[5]}$ and in widely different organisms, ${ }^{[6]}$ requires to urgently identify and develop at industrial level new antimicrobial solutions against said pathogens.
To identify the targets for future antibacterials capable of preventing biofilm formation on indwelling plastic tubes frequently used in clinical settings, scholars in India recently identified the 56 specific proteins in highly virulent $P$. aeruginosa PA14 strain regulating its ability to form biofilm on said tubes. ${ }^{[7]}$

In general, to manage Pseudomonas aeruginosa infections, causing high mortality in critically ill and immunocompromised patients driven by the appearance of drug-resistant strains, today's therapeutic options include antibiotic combinations based on pharmacokinetic and pharmacodynamic analyses. ${ }^{[8]}$

Highly desirable new anti-pseudomonal medicines should not drive superinfection, ${ }^{[8]}$ and be preferably available as oral formulations to allow step-down therapy in the treatment of Gram-negative bloodstream infection. ${ }^{[0]}$

${ }^{[a]} \mathrm{Dr}$. A. Presentato, Prof. R. Alduina

Department of Biological, Chemical and Pharmaceutical Sciences and Technologies, University of Palermo, viale delle Scienze, 90028 Palermo (Italy)

E-mail: valeria.alduina@unipa.it

${ }^{[b]}$ Dr. A. Scurria, Dr. M. Pagliaro, Dr. R. Ciriminna Istituto per lo Studio dei Materiali Nanostrutturati, CNR via U. La Malfa 153

90146 Palermo (Italy)

E-mail: rosaria.ciriminna@cnr.it

${ }^{[c]}$ Dr. L. Albanese, Dr. F. Zabini, Dr. F. Meneguzzo Istituto per la Bioeconomia, CNR via Madonna del Piano 10 50019 Sesto Fiorentino FI (Italy)

${ }^{[d]}$ Dr. D. Nuzzo, Dr. P. Picone

Istituto per la Ricerca e l'Innovazione Biomedica, CNR via U. La Malfa 153 90146 Palermo (Italy)

E-mail: domenico.nuzzo@irib.cnr.it
Similarly, Escherichia coli is a versatile Gram-negative bacterial strain colonizing the human gastrointestinal tract, where it becomes part of the microbiota a few hours after birth. Specific pathotypes of $E$. coli may infect healthy individuals affording three possible syndromes: enteric disease and diarrhea, urinary tract infection, and meningitis. ${ }^{[10]}$

In a previous study, lemon IntegroPectin was shown to exert significant activity in vitro against Staphylococcus aureus virulent strains. ${ }^{[1]}$ We now report the discovery of high in vitro activity of lemon IntegroPectin against virulent strains of $P$. aeruginosa, evaluating its antibacterial effect in terms of the minimal bactericidal (MBC) and minimal inhibitory concentration (MIC). We also show that this new biomaterial inhibits $E$. coli proliferation by exerting a powerful oxidative stress action against the microorganism.

\section{Results and Discussion}

Lemon IntegroPectin (IntPec) is effectively and reproducibly produced via hydrodynamic cavitation of waste lemon peel in water only. The lemon peel was derived by an in-line extractor at 
a lemon juice factory and directly processed on semi-industrial scale (34 kg of waste lemon peel of organically grown Siracusa lemons in $120 \mathrm{~L}$ tap water) using the same cavitation conditions lately developed to process waste orange peel. ${ }^{[12]}$

Easily dissolved in water, the resulting yellow pectin flakes obtained after freeze drying have a pleasant lemon smell and are completely devoid of cytotoxic activity against human pulmonary cells up to high concentration $(1 \mathrm{mg} / \mathrm{mL}) .{ }^{[13]}$ Furthermore, lemon IntPec has an extremely high content of polyphenols adsorbed at its surface: $0.88 \mathrm{mg} \mathrm{GAE} / \mathrm{g}$ (in terms of gallic acid equivalents, or GAE, per dry gram of pectin) versus $8.3 \times 10^{-3} \mathrm{mg} \mathrm{GAE} / \mathrm{g}$ for the lemon peel of the cultivar with the highest biophenol concentration.

We tested the antimicrobial activities of IntPec against $P$. aeruginosa 10145 strain (indicator pathogen strain) commonly used as a positive control for molecular detection in bioaerosols, as well as a quality control strain for drugs. ${ }^{[14]}$ For comparison, we also tested the antibacterial activity of commercial citrus pectin (galacturonic acid $\geq 74.0 \%$, dry basis, from Merck Life Science, Milan, Italy).

Figure 1 shows evidence that both commercial pectin and IntPec inhibit $P$. aeruginosa growth. However, the number of viable cells decreased by $2.6 \mathrm{log}$ units when the concentration of IntPec went from 0 (control) to $10 \mathrm{mg} \mathrm{mL}^{-1}$, whereas it remained almost unvaried (from 9.1 to 9.0 ) when the concentration of commercial citrus pectin was $10 \mathrm{mg} \mathrm{mL}^{-1}$.

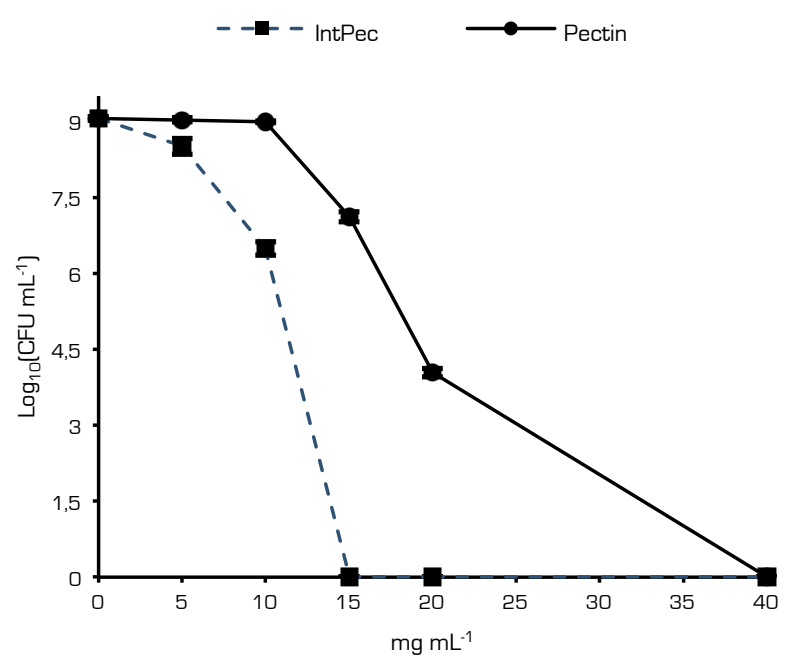

Figure 1. Viable cell count of $P$. aeruginosa 10145 strain after incubation in culture broth added with increasing concentrations of IntPec and commercial citrus pectin.

Increasing the concentration of IntegroPectin from 10 to just 15 $\mathrm{mg} \mathrm{mL}^{-1}$, the $\log _{10}(\mathrm{CFU})$ reached the 0 value, obtaining a total killing effect and establishing the MBC of the lemon IntegroPectin against $P$. aeruginosa. At the same concentration of commercial citrus pectin, the $\log _{10}$ (CFU) was still 7.1. A concentration of $40 \mathrm{mg} \mathrm{mL}^{-1}$ of commercial pectin was required to observe $\log _{10}$ (CFU) to reach the 0 value.

Evidence of the reduction of viable $P$. aeruginosa 10145 CFUs challenged in vitro with pectin and IntPec is displayed in Figure 2.
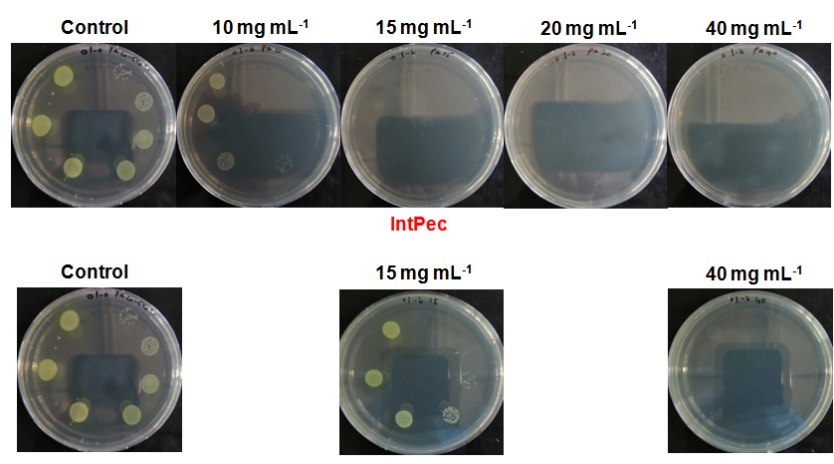

Figure 2. Evidence of in vitro pectin and IntegroPectin activity against $P$. aeruginosa 10145 strains.

To establish MIC values of both pectins, we measured the optical density at $600 \mathrm{~nm}$ (at this wavelength the bacterial cells are not harmed whereas light scattered by the cells no longer reaches the photoelectric cell translating into higher turbidity) of the challenged $P$. aeruginosa cultures as compared to unchallenged ones. We briefly remind that the MIC value is defined as the lowest concentration of an antimicrobial agent that prevents visible growth of a microorganism in a broth dilution susceptibility test. ${ }^{[15]}$

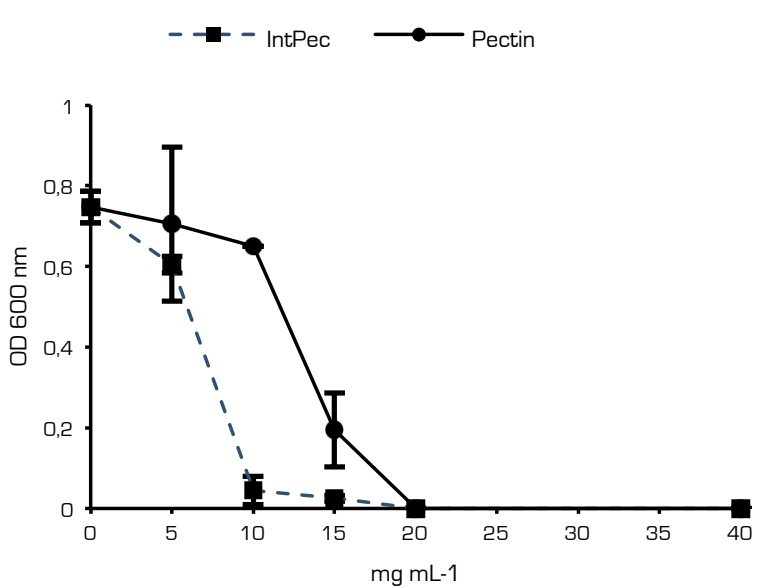

Figure 3. Optical density at $660 \mathrm{~nm}$ of $P$. aeruginosa ATCC 10145 cultures in the presence of lemon IntPec and of commercial citrus pectin for the assessment of minimal inhibitory concentration (MIC).

Figure 3 shows that the MIC value of lemon IntPec is $10 \mathrm{mg} \mathrm{mL}^{-}$ ${ }^{1}$ whereas that of commercial citrus pectin is $20 \mathrm{mg} \mathrm{mL}^{-1}$. 
To better understand the relevance of these results, it is instructive to realize that the recommended broth dilution method to determine the minimal inhibitory concentration of

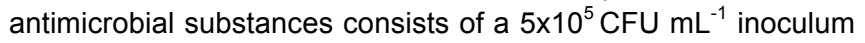
size ${ }^{[16]}$ while in the present study on the efficacy of lemon IntPec against $P$. aeruginosa, the broth was inoculated with a cellular load 2 orders of magnitude higher than the recommended bacterial concentration.

To provide preliminary insight on the antibacterial mechanism, the effect of IntPec was also tested on Escherichia coli growth by incubating cells in presence of sub-inhibitory concentrations of IntPec.

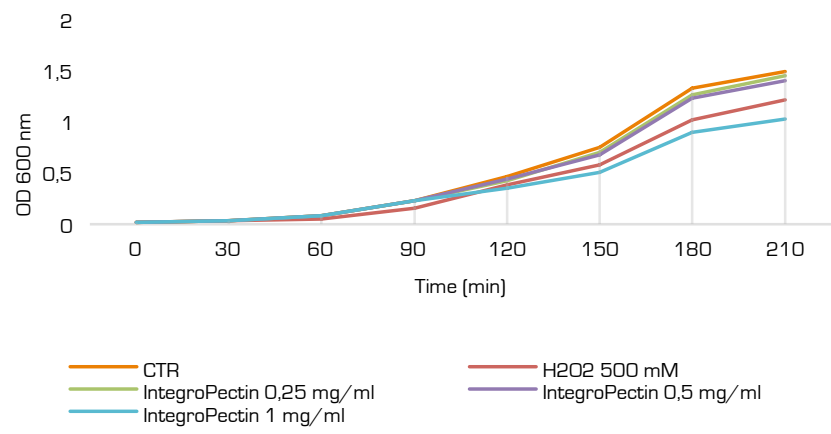

Figure 4. Effect of $\mathrm{H}_{2} \mathrm{O}_{2} 500 \mathrm{mM}$ and of lemon IntPec increasing concentrations on $E$. Coli proliferation. CTR stands for control sample.

Figure 4 shows evidence of the increasing antibacterial activity of IntPec at increasing concentrations.

While little activity is observed at $0.25 \mathrm{mg} / \mathrm{mL}$ titre, it is enough to add the lemon IntPec at $1.0 \mathrm{mg} / \mathrm{mL}$ concentration to observe a dramatic reduction in the $E$. coli cell proliferation, significantly higher than that caused by concentrated $(0.5 \mathrm{M})$ aqueous hydrogen peroxide $\left(\mathrm{H}_{2} \mathrm{O}_{2}\right)$, a powerful oxidizing agent that at this concentration is capable to quickly denature enzymes and oxidize not only protein side chains but also the protein backbone. ${ }^{[17]}$

To evaluate the possible generation of Reactive Oxygen Species (ROS), E. coli bacteria were incubated with a diluted solution of dichlorodihydrofluorescein diacetate (DCFH-DA), the most widely used probe for detecting intracellular $\mathrm{H}_{2} \mathrm{O}_{2}$ and oxidative stress, ${ }^{[18]}$ alone or in the presence of increasing concentrations of IntPec.

Figure 5 shows that already at $0.5 \mathrm{mg} / \mathrm{mL}$ concentration the bioproduct exerts a powerful oxidative stress on the bacteria, significantly higher than $\mathrm{H}_{2} \mathrm{O}_{2} \quad 0.5 \mathrm{M}$. When the IntPec concentration is increased to $1.0 \mathrm{mg} / \mathrm{mL}$, the ROS generation becomes more than twice higher than that driven by concentrated $\mathrm{H}_{2} \mathrm{O}_{2}$.

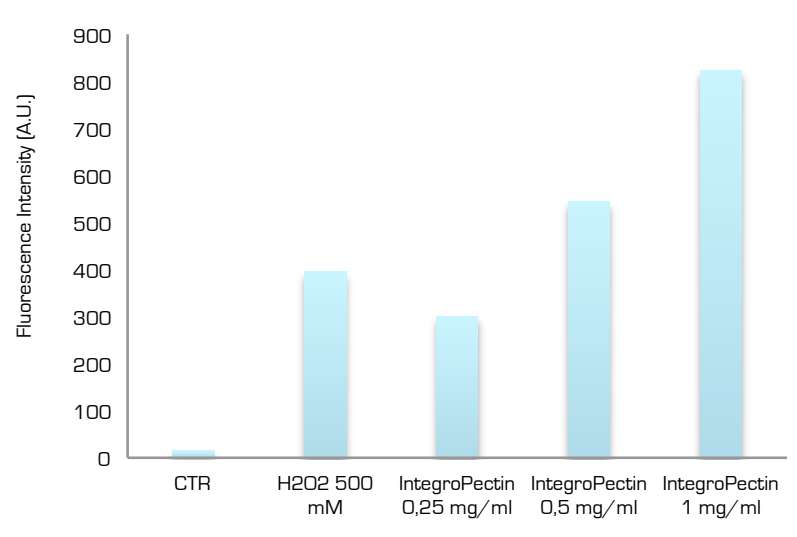

Figure 5. Generation of ROS on $E$. coli bacteria driven by aqueous $\mathrm{H}_{2} \mathrm{O}_{2}$ $0.5 \mathrm{M}$ and by increasing concentrations of lemon IntPec. CTR stands for control sample.

Figure 6 shows the quick production of ROS estimated again using DCFH-DA after increasing the IntPec concentration from $0.25 \mathrm{mg} / \mathrm{mL}$ to $1 \mathrm{mg} / \mathrm{mL}$. The amount of ROS formed at the surface of $E$. coli follows an almost identical profile for $\mathrm{H}_{2} \mathrm{O}_{2}$ $500 \mathrm{mM}$ and aqueous $\mathrm{IntPec}$ at $0.5 \mathrm{mg} / \mathrm{mL}$ concentration.

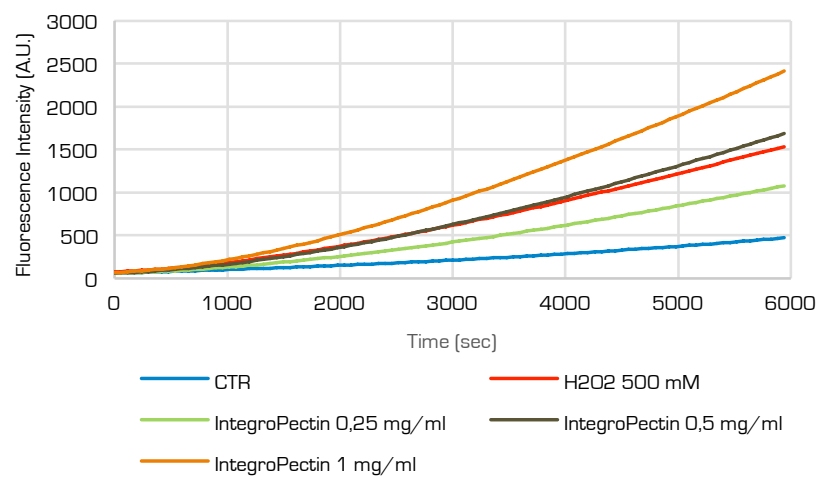

Figure 6. $E$. coli oxidation kinetics driven by by aqueous $\mathrm{H}_{2} \mathrm{O}_{2} 0.5 \mathrm{M}$ and by increasing concentrations of lemon IntPec. CTR stands for control sample.

Yet, using lemon IntPec at $1.0 \mathrm{mg} / \mathrm{mL}$ already after $16 \mathrm{~min}$ $(\sim 1000 \mathrm{~s})$ the amount of ROS is higher than that generated by concentrated hydrogen peroxide, to eventually almost double the oxidative stress exerted by $\mathrm{H}_{2} \mathrm{O}_{2} 0.5 \mathrm{M}$ after $1.7 \mathrm{~h}$ since the addition of the new pectic substance.

The same lemon IntPec was recently shown to exert significant activity in vitro against $S$. aureus virulent strains. ${ }^{[11]}$ It is therefore of direct relevance to this account the fact that Gram-positive $S$. aureus is often present in conjunction to $P$. aeruginosa forming hazardous polymicrobial complex communities particularly resistant to antibiotics. ${ }^{[19]}$ 
Originally reported in the late $1930 \mathrm{~s},{ }^{[20]}$ the antibacterial properties of pectin were rediscovered in the late 1990s by scholars in Russia. Pectin, usually derived from citrus peel or apple pomace, was found to be the only food fiber showing bactericidal activity on the most widely distributed pathogenic and opportunistic microorganisms, decreasing the need for antimicrobial penicillins. ${ }^{[21]}$

In 2015, scholars in Lebanon reported the antibacterial activity of citrus pectin against $S$. aureus, with optimal antibacterial activity observed at $\mathrm{pH} 6$ with MIC values ranging from $0.39 \mathrm{mg} \mathrm{mL}^{-1}$ to $3.125 \mathrm{mg} \mathrm{mL}^{-1} \cdot{ }^{[22]}$ However, scholars in Taiwan in 2014 reported lack of any antibacterial activity of citrus pectin against $P$. aeruginosa. ${ }^{[23]}$

We make the hypothesis that the antibacterial action of lemon IntegroPectin is due to a synergistic combination of $i$ ) the pectic polymer, ii) the lemon oil adsorbed at its surface (the citrus essential oil is present in the form of a highly stable nanoemulsion in the aqueous phase after the hydrocavitation process), ${ }^{[12]}$ and iii) the lemon flavonoids highly concentrated (with respect to the citrus peel) at the surface of lyophilized IntPec. ${ }^{[13]}$

Likewise to other citrus oils, lemon oil is particularly active against Gram-positive bacteria, even though selected Gramnegative bacteria such as $E$. coli and Campylobacter jejuni are inhibited by lemon oil due to the presence of oxygenated monoterpenes exhibiting much stronger antimicrobial activity than hydrocarbon monoterpenes present in the essential oil. ${ }^{[24]}$

These results were subsequently confirmed for the fungicidal action of lemon oil against Candida albicans pathogenic yeasts, wherein the most active antimicrobial was found to be citral. ${ }^{[25]}$ Remarkably, the concentration of the latter oxygenated monoterpene is particularly abundant in the peel of citrus grown (in Sicily) according to organic farming principles. ${ }^{[26]}$ Indeed, the waste lemon peel used to produce the IntPec was obtained by a citrus company in Sicily using only organically grown lemons.

Finally, citrus flavonoids chiefly contained in the flavedo and in the albedo are well known antibacterials. ${ }^{[27]}$ Only recently the mechanism through which they inhibit $P$. aeruginosa biofilm formation was identified. Flavonoids possessing dihydroxyl moieties in the flavone A-ring backbone prevent LasR DNA binding due to the inhibitory action of the two hydroxyl groups, with one of them that must be at position 7 on the A-ring for potent inhibition of the aforementioned binding. ${ }^{[28]}$

In doing so, citrus flavonoids inhibit quorum sensing (QS, the bacterial cell-cell communication process controlling collective behavior) via antagonism of the QS receptor, the LasR protein. The latter protein indeed is a major transcriptional activator of $P$. aeruginosa QS and plays a pivotal role in the activation of many virulence genes. ${ }^{[29]}$

Administration of citrus flavonoids to $P$. aeruginosa alters transcription of quorum sensing-controlled target promoters and suppresses virulence factor production, confirming their potential as anti-infectives that do not function by means of traditional bactericidal or bacteriostatic mechanisms. ${ }^{[28]}$

All main lemon flavonoids, hesperidin (hesperetin 7-O-betarutinoside), eriocitrin (eriodictyol 7-O-beta-rutinoside), and diosmin (diosmetin 7-O-rutinoside) are the glycosides of flavonoids of similar compounds bearing two dihydroxyl moieties in the flavone A-ring backbone, with the hydroxyl at position 7 on the A-ring derivatised with a sugar. ${ }^{[30]}$ The latter glycosidic bond is easily hydrolyzed at the bacterial membrane level in the aqueous culture broth.

Accordingly, orange extracts rich in glycosylated flavanones including hesperidin, naringenin, and naringin are known to effectively interfere and inhibit quorum sensing in $P$. aeruginosa $^{[31]}$ as well as in Gram-negative Yersinia enterocolitica ${ }^{[32]}$ bacteria.

\section{Conclusions}

In conclusion, we have discovered the powerful in vitro activity of lemon IntegroPectin against virulent strains of $P$. aeruginosa and E. coli. Aiming to identify the mechanism of action of this novel bioproduct, we have also discovered that dissolved in solution at the concentration of $1 \mathrm{mg} \mathrm{mL}^{-1}$, it exerts an oxidative stress action against the latter microorganism that is more than twice stronger than concentrated $(0.5 \mathrm{M})$ hydrogen peroxide.

Taking into account the complete lack of cytotoxicity of citrus IntPec against human lung cells at the aforementioned concentration $\left(1 \mathrm{mg} \mathrm{mL}^{-1}\right),{ }^{[13]}$ and the antibacterial activity of lemon IntPec against Gram-positive $S$. aureus, ${ }^{[1]}$ these results may open the route to the industrial development a new antibacterial treatment against polymicrobial infections based on a new bioproduct reproducibly and economically obtained in large amounts from the main citrus industry's by-product.

\section{Experimental}

\section{Experiments with $P$. aeruginosa}

The antibacterial activity of IntePec and commercial citrus pectin was preliminarily assessed through the determination of MBC and MIC. Specifically, stationary grown cells of $P$. aeruginosa were inoculated $\left(1.19 \times 10^{7} \pm 8.01 \times 10^{5} \mathrm{CFUmL}^{-1} n=6\right)$ in Luria Bertani medium (hereafter named as LB and composed of $\left[\mathrm{g} \mathrm{L}^{-1}\right]$ sodium chloride [10], trypton [10], and yeast extract [5]) amended with increasing concentrations (i.e., 5, 10, 15, 20, and $40 \mathrm{mg} \mathrm{mL}^{-1}$ ) of either lemon IntPec or commercial citrus pectin, being then challenged for $24 \mathrm{~h}$ at $37^{\circ} \mathrm{C}$ under mechanical shacking (180 rpm).

Non-challenged bacterial cultures were incubated under the same conditions and used as a control. After $24 \mathrm{~h}$ challenge, all the bacterial cultures were serially diluted and aliquots $(20 \mu \mathrm{L})$ of the diluted cultures, as well as the undiluted ones, were spotted onto LB agar $\left(15 \mathrm{~g} \mathrm{~L}^{-1}\right)$ plates and recovered at $37^{\circ} \mathrm{C}$ under static mode. ${ }^{[33]}$ The kill curve reporting the number of viable CFU $\mathrm{mL}^{-1}$ as a function of the concentration of both pectins is 
expressed in logarithmic $\left(\log _{10}\right)$ scale with standard deviation ( $n$ $=3$ ), as described elsewhere. ${ }^{[34]}$

Experiments with E. coli

A single colony was inoculated into LB medium and incubated at $37^{\circ} \mathrm{C}$ overnight (o.n.). An aliquot $(5 \mu \mathrm{L})$ of the o.n. bacterial culture, approximately $10^{9} \mathrm{CFU} / \mathrm{mL}$, was added to three test tubes containing fresh LB medium $(5 \mathrm{~mL})$. IntPec $(0.25,0.5$ and $1 \mathrm{mg} / \mathrm{mL}$ ) was added separately to the culture medium at time $0 \mathrm{~min}$ or $210 \mathrm{~min}$. The growth was determined by reading the absorbance value at $600 \mathrm{~nm}$ (OD600) using a Spark $10 \mathrm{M}$ multimode microplate reader (Tecan Group, Männedorf, Switzerland) with $30 \mathrm{~min}$ intervals.

For ROS generation, an aliquot of the $E$. coli o.n. culture, approximately $10^{9} \mathrm{CFU} / \mathrm{mL}$, was diluted $\left(1: 10^{5}\right)$ and a $100 \mu \mathrm{L}$ sample placed in a 96-well optical bottom white microplate. IntPec at different concentration $(0.25,0.5$ and $1 \mathrm{mg} / \mathrm{mL})$ was added to the wells. Then, the samples were incubated with $1 \mathrm{mM}$ of DCFH-DA (Molecular Probes, Eugene, OR, USA) for $8 \mathrm{~h}$ at $37^{\circ} \mathrm{C}$. Afterward, the $E$. coli samples were analyzed by using a GloMax Microplate Reader (Promega, Madison, WI, USA) for fluorescence detection at the excitation wavelength of $475 \mathrm{~nm}$ and emission wavelength $555 \mathrm{~nm}$. An untreated $E$. coli bacterial culture (CTR) and an E. coli culture challenged with $500 \mathrm{mM}$ $\mathrm{H}_{2} \mathrm{O}_{2}$ were used as control for growth and oxidative stress.

For oxidation kinetics, an aliquot of $E$. coli o.n. culture, approximately $10^{9} \mathrm{CFU} / \mathrm{mL}$, was diluted $\left(1: 10^{5}\right)$ and a $100 \mu \mathrm{L}$ sample placed in a 96-well optical bottom white microplate. IntPec at different concentration $(0.25,0.5$ and $1 \mathrm{mg} / \mathrm{mL})$ was added to the wells. The production of ROS was estimated using DCFH-DA at a final concentration of $1 \mathrm{mM}$. Untreated $E$. coli (CTR) was used as control and aqueous $\mathrm{H}_{2} \mathrm{O}_{2} 0.5 \mathrm{M}$ was used as a positive control of oxidation state. The oxidation kinetics was followed by fluorescence at the excitation wavelength of $475 \mathrm{~nm}$ and emission wavelength $555 \mathrm{~nm}$ by a GloMax Discover System (Promega, Madison, WI, USA) in a 96-multiwell plate incubated for $2 \mathrm{~h}$ at $37^{\circ} \mathrm{C}$.

\section{Acknowledgements}

Thanks to Campisi Italia (Siracusa, Italy) for the gift of waste lemon peel from which the IntegroPectin used in this study was obtained. We thank Dr M. Di Carlo, CNR-IRIB, for purposeful collaboration.

\section{Author Information}

\section{ORCID}

Alessandro Presentato: 0000-0002-4794-0599

Antonino Scurria: 0000-0001-5624-6833

Lorenzo Albanese:

Pasquale Picone: 0000-0001-7127-2183

Mario Pagliaro: 0000-0002-5096-329X

Federica Zabini: 0000-0003-1505-0839
Francesco Meneguzzo: 0000-0002-5952-9166

Rosa Alduina: 0000-0003-1054-6915

Domenico Nuzzo: 0000-0002-4325-417X

Rosaria Ciriminna: 0000-0001-6596-1572

\section{Notes}

The Authors declare no conflict of interest

Keywords: Pseudomonas aeruginosa; polymicrobial infections; IntegroPectin; flavonoids; antibacterial

\section{References}

[1] A. Presentato, S. Lampis, A. Vantini, F. Manea, F. Daprà, S. Zuccoli, G. Vallini, On the ability of Perfluorohexane Sulfonate (PFHxS) bioaccumulation by two Pseudomonas sp. strains isolated from PFAS-contaminated environmental matrices, Microorganisms 2020, 8, 92.

[2] H. Mikkelsen, R. McMullan, A. Filloux, The Pseudomonas aeruginosa Reference Strain PA14 Displays Increased Virulence Due to a Mutation in ladS, PLoS One 2011, 6:e29113.

[3] J. L. Lamas Ferreiro, J. Alvarez Otero, L. Gonzalez Gonzalez, L. Novoa Lamazares, A. Arca Blanco, J. R. Bermudez Sanjurjo, I. Rodriguez Conde, M. Fernandez Soneira, J. de la Fuente Aguado, Pseudomonas aeruginosa urinary tract infections in hospitalized patients: mortality and prognostic factors, PLOS One 2017, 12:e0178178.

[4] S. Malhotra, D. Hayes Jr., D. J. Wozniak, Cystic Fibrosis and Pseudomonas aeruginosa: the Host-Microbe Interface, Clin. Microbiol. Rev. 2019, 32: e00138-18.

[5] R. Alduina, Antibiotics and Environment, Antibiotics 2020, 9, 202.

[6] M. F. Blasi, L. Migliore, D. Mattei, A. Rotini, M. C. Thaller, R. Alduina Antibiotic Resistance of Gram-Negative Bacteria from Wild Captured Loggerhead Sea Turtles, Antibiotics 2020, 9, 162

[7] D. Badal, A. V. Jayarani, M. A. Kollaran, A. Kumar, V. Singh, Pseudomonas aeruginosa biofilm formation on endotracheal tubes requires multiple two-component systems, J. Med. Microbiol. 2020, DOI: 10.1099/jmm.0.001199

[8] M. Bassetti, A. Vena, A. Croxatto, E. Righi, B. Guery, How to manage Pseudomonas aeruginosa infections, Drugs Context 2018, 7:212527.

[9] S. A. Nisly, D. L. McClain, A. G. Fillius, K. A. Davis, Oral antibiotics for the treatment of Gram-negative bloodstream infections: A retrospective comparison of three antibiotic classes, J. Glob. Antimicrob. Resist. 2020, 20, 74.

[10] S. A. Nisly, D. L. McClain, A. G. Fillius, K. A. Davis, Pathogenic Escherichia coli, Nat. Rev. Microbiol. 2004, 2, 123.

[11] A. Presentato, A. Scurria, L. Albanese, C. Lino, M. Sciortino, M.Pagliaro, F. Zabini, F. Meneguzzo, R. Alduina, D. Nuzzo, R. Ciriminna, Superior antibacterial activity of integral lemon pectin from hydrodynamic cavitation, ChemistryOpen 2020, 9, 628.

[12] F. Meneguzzo, C. Brunetti, A. Fidalgo, R. Ciriminna, R. Delisi, L. Albanese, F. Zabini, A. Gori, L. B. dos Santos Nascimento, A. De Carlo, F. Ferrini, L. M. Ilharco, M. Pagliaro, Real-Scale Integral Valorization of Waste Orange Peel via Hydrodynamic Cavitation, Processes 2019, 7, 581.

[13] D. Nuzzo, L. Cristaldi, M. Sciortino, L. Albanese, A. Scurria, F. Zabini, C.Lino, M. Pagliaro, F. Meneguzzo, M. Di Carlo, R. Ciriminna, Exceptional antioxidant, non-cytotoxic activity of integral lemon pectin from hydrodynamic cavitation, ChemistrySelect 2020, 5, 5066. 
[14] American Type Culture Collection, Pseudomonas aeruginosa (ATCC $® 10145^{\mathrm{TM}}$ ), 2020. Available at the URL: www.atcc.org

[15] Clinical and Laboratory Standards Institute, Methods for dilution antimicrobial susceptibility tests for bacteria that grow aerobically, approved standard, $10^{\text {th }}$ edition, Wayne, PA: 2015.

[16] I. Wiegand, K. Hilpert, R. E. W. Hancock, Agar and broth dilution methods to determine the minimal inhibitory concentration (MIC) of antimicrobial subtances, Nat. Protoc. 2008, 3, 163.

[17] M. Finnegan, E. Linley, S. P. Denyer, G. McDonnell, C. Simons, J.-Y. Maillard, Mode of action of hydrogen peroxide and other oxidizing agents: differences between liquid and gas forms, J Antimicrob Chemother. 2010, 65, 2108.

[18] B. Kalyanaraman, V. Darley-Usmar, K. J. A. Davies, P. A. Dennery, H. J. Forman, M. B. Grisham, G. E. Mann, K. Moore, L. Jackson Roberts, II, H. Ischiropoulos, Measuring reactive oxygen and nitrogen species with fluorescent probes: challenges and limitations, Free Radic Biol Med. 2012, 52, 1.

[19] T. P. Cushnie, A. J. Lamb, Pseudomonas aeruginosa and Staphylococcus aureus Communication in Biofilm Infections: Insights Through Network and Database Construction, Crit. Rev. Microbiol. 2019, 45, 712.

[20] E. Hayxes, C. A. Tompkins, G. Washburn, M. Winter Bactericidal Action of Pectin, Exp. Biol. Med. 1937, 36, 839.

[21] D. D. Men'shikov, E. B. Lazareva, T. S. Popova, L. U. Shramko, I. S. Tokaev, G. V. Zalogueva, I. N. Gaponova, Antimicrobial properties of pectins and their influence on antibiotic activity, Antibiot. Khimioter. 1997, 42, 10.

[22] R. M. Abdel-Massih, V. Hawach, Z. Daoud, Antibacterial and anti-proliferative activity of different forms of Citrus pectin, $J$. Nutr. Food Sci. 2015, 5, 4.

[23] M.-C. Wu, H.-C. Li, P.-H. Wu, P.-H. Huang, Y.-T. Wang, Assessment of Oligogalacturonide from Citrus Pectin as a Potential Antibacterial Agent against Foodborne Pathogens, J. Food Sci. 2014, 79: M1541.

[24] L. Settanni, E. Palazzolo, V. Guarrasi, A. Aleo, C. Mammina, G. Moschetti, M. A. Germanà, Inhibition of foodborne pathogen bacteria by essential oils extracted from citrus fruits cultivated in Sicily, Food Control 2012, 26, 326.

[25] M. Białoń, T. Krzyśko-Łupicka, M. Koszałkowska, P. P. Wieczorek, The Influence of Chemical Composition of Commercial Lemon Essential Oils on the Growth of Candida Strains, Mycopathologia 2014, 177, 29.

[26] A. Verzera, A. Trozzi, G. Dugo, G. Di Bella, A. Cotroneo, Biological lemon and sweet orange essential oil composition, Flavour Fragrance J. 2004, 19, 544.

[27] F. Farhadi, B. Khameneh, M. Iranshahi, M. Iranshahy, Antibacterial activity of flavonoids and their structure-activity relationship: An update review, Phytother. Res. 2019, 33, 13.

[28] J. E. Paczkowski, S. Mukherjee, A. R. McCready, J.-P. Cong, C. J. Aquino, H. Kim, B. R. Henke, C. D. Smith, B. L. Bassler, Flavonoids Suppress Pseudomonas aeruginosa Virulence through Allosteric Inhibition of Quorum-sensing Receptors, J. Biol. Chem. 2017, 292, 4064.

[29] N. Chowdhury, A. Bagchi, Molecular insight into the activity of LasR protein from Pseudomonas aeruginosa in the regulation of virulence gene expression by this organism, Gene 2016, $580,80$.

[30] J. A. Del Río, M. D.Fuster, P. Gómez, I. Porras, A. GarcíaLidón, A. Ortuño, Citrus limon: a source of flavonoids of pharmaceutical interest, Food Chem. 2004, 84, 457.

[31] O. M. Vandeputte, M. Kiendrebeogo, T. Rasamiravaka, C. Stevigny, P. Duez, S. Rajaonson, The flavanone naringenin reduces the production of quorum sensing-controlled virulence factors in Pseudomonas aeruginosa PAO1, Microbiology 2011, $157,2120$.

[32] P. Truchado, J. A. Gimenez-Bastida, M. Larrosa, I. CastroIbanez, J. C. Espin, F. A. Tomas-Barberan, Inhibition of quorum sensing (QS) in Yersinia enterocolitica by an orange extract rich in glycosylated flavanones, J. Agric. Food Chem. 2012, 60, 8885.

[33] A. Presentato, E. Piacenza, A. Darbandi, M. Anikovskiy, M. Cappelletti, D. Zannoni, R. J. Turner, Assembly, growth and conductive properties of tellurium nanorods produced by Rhodococcus aetherivorans BCP1, Sci. Rep. 2018, 8, Article number: 3923.

[34] (a) E. Piacenza, A. Presentato, E. Ambrosi, A. Speghini, R. J. Turner, G. Vallini, S. Lampis, Physical-chemical properties of biogenic selenium nanostructures produced by Stenotrophomonas maltophilia SeITE02 and Ochrobactrum sp. MPV1, Front. Microbiol. 2018, 9, 3178; (b) M. V. Raimondi, A. Presentato, G. Li Petri, M. Buttacavoli, A. Ribaudo, V. De Caro, R. Alduina, P. Cancemi, New Synthetic Nitro-Pyrrolomycins as Promising Antibacterial and Anticancer Agents, Antibiotics 2020, 9, 292. 
Towards a new treatment against polymicrobial infections: High antibacterial activity of lemon IntegroPectin against Pseudomonas aeruginosa and Escherichia coli

A. Presentato, A. Scurria, L. Albanese, P. Picone, M. Pagliaro, F. Zabini, F. Meneguzzo, R. Alduina, D. Nuzzo, R. Ciriminna
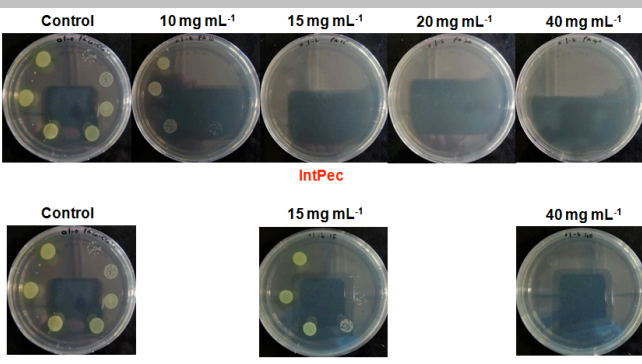

Pectin

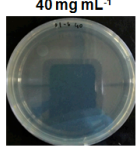

